Ann. Zootech., 1975, 24 (2), I77-I87.

\author{
I.N.R.A. A.
BIBLIOTHEQUE UO 35905
DOMAINE OE CROUELLE \\ 63039 \\ CLERMONT-FD CEDEX 2
}

\title{
AIRES DE RÉSIDENCE PRÉFÉRENTIELLES D'UN GROUPE DE VACHES LAITIERES EN STABULATION LIBRE
}

\author{
G. THINÈs, Monique SOFFIÉ et G. de MARNEFFE \\ Centre de Psychologie expérimentale et comparée, \\ Université de Louvain, \\ B-3041 Pellenberg (Belgique)
}

\section{RÉSUMÉ}

Les observations analysées dans ce travail ont porté sur des vaches laitières et ont été réaliséos dans une exploitation utilisant le système de stabulation libre. Celui-ci comportait une aire extérieure cimentée en communication permanente avec une étable dans laquelle les animaux avaient libre accès à des stalles individuelles. Les résultats obtenus sur une population comprenant 37 vaches et un taureau, permettent les conclusions suivantes :

I ${ }^{0}$ La répartition des animaux dans l'étable est systématique, chaque animal manifestant une préférence significative pour une stalle particulière.

$2^{\circ}$ En ce qui concerne la fréquentation de l'aire extérieure, celle-ci est assez homogène. Cependant, on constate qu'une zone particulière est occupée plus souvent par l'ensemble du troupeau et que certains animaux manifestent des préférences locales marquées.

On peut supposer que le choix des stalles préférentielles est guidé, au moins à titre partiel, par une perception configurationnelle de l'aire totale disponible, sans qu'il soit possible d'établir, sur la base des observations effectuées à ce jour, quels signaux sensoriels seraient responsables de ce comportement. Dans l'aire extérieure, il existe une zone préférentielle liée aux comportements de communication et un territoire d'alimentation. discutée.

La légitimité de la notion de territoire à propos des répartitions spatiales observées, est

\section{INTRODUCTION}

La territorialité est un phénomène qui n'a guère été étudié chez les bovins domestiques. Les quelques travaux qui ont abordé ce problème ont surtout considéré les préférences locales, la répartition et les déplacements d'animaux en pâture, 
situation dans laquelle le type d'herbage, sa densité ainsi que le rang social de l'animal jouent le rôle principal. En effet, les distances couvertes sont moins importantes lorsque la pâture est encore intacte; en outre les déplacements et la présence d'un animal en un endroit particulier peut être liée à la localisation élective de certaines plantes et, dans certains cas, à la présence d'un être humain (BEILHARZ et MYLREA, I963 ; HANCOCK, I953 ; VoIsIN, I959; TrIBE, I955). Par ailleurs, en ce qui concerne les bovins sauvages, des comportements territoriaux ont été observés dans les aires de pâture et d'abreuvage ainsi que dans les lieux fréquentés préférentiellement par les animaux pour le repos, les comportements de confort et les interactions sociales (SCHLOETH, I96I).

Quant à nos observations elles ont été effectuées dans une exploitation pratiquant un régime de stabulation libre, les animaux ayant accès en permanence d'une aire extérieure (parc) à une étable et inversement. Dans ces conditions, qui étaient fort éloignées tant du régime de pâture que du régime sauvage, nous avons étudié la répartition spatiale d'un groupe de vaches laitières dans les deux aires précitées. Concrètement, il s'agissait d'examiner si dans un tel type d'exploitation, les vaches occupaient l'espace disponible de façon aléatoire ou si elles choisissaient au contraire certaines aires de résidence de façon systématique.

\section{MATÉRIEL E'T MÉTHODE}

Nos observations ont porté sur un groupe composé de 37 vaches laitières et d'un taur eau Toutes les vaches étaient de race Pie noive hollandaise à l'exception de quatre Pie bleue de moyenne Belgique et de deux canadiennes Holstein. Le Taureau était un Holstein pur. L'âge des animaux était compris entre 4 et 8 ans. Les animaux en période d'œstrus ont été exclus des résultats. Tous les animaux portaient un collier muni d'une plaque numérotée (dimension des chiffres : $6 \times 2 \mathrm{~cm}$ ). Les observations, tant dans l'étable que dans le parc extérieur, ont été effectuées alternativement par deux observateurs dont on avait au préalable vérifié l'équivalence en comparant des données obtenues simultanément. Les résultats ont tous été notés directement. Seuls les contacts agonistiques observés lors des épreuves de compétition de nourriture ont été enregistrés sur bande magnétique.

\section{A. - Observations dans l'étable}

Les animaux occupant une place individuelle à l'intérieur de l'étable (fig. I) il était facile de relever leurs positions avec précision. Il faut souligner que les 37 animaux du troupeau disposaient d'un total de jo stalles. Chaque animal pouvait donc choisir toute une série de lieux de couchage sans être obligé de déloger un congénère. Cette situation relativement exceptionnelle permettait donc de mettre en évidence les préférences libres des animaux du fait que la contrainte spatiale y était faible. Nos observations s'étendent du 28 février au I 2 juin 1973. Le relevé des positions occupées par les animaux s'effectuait à une heure où la majorité de ceux-ci était dans l'étable c'est-à-dire avant le lever ou après le coucher du soleil. Les locaux n'étant pas éclairés la nuit, le repérage des animaux s'effectuait au moyen d'une torche à main. Nous n'avons pris en considération qu'une seule observation par nuit et nous n'avons tenu compte que des positions occupées par les animaux pratiquement présents de façon permanente, à l'exclusion de ceux qui n'étaient introduits que temporairement dans le troupeau.

\section{B. - Observations dans le parc extérieur}

Ces observations s'étendent du 5 mai au 16 juin 1973 et portent sur les mêmes animaux. Nous avions délimité quatre aires fictives de superficie approximativement équivalentes (A, B, C et D), les limites de celles-ci étant constituées par les clôtures extérieures du parc et les côtés de la 


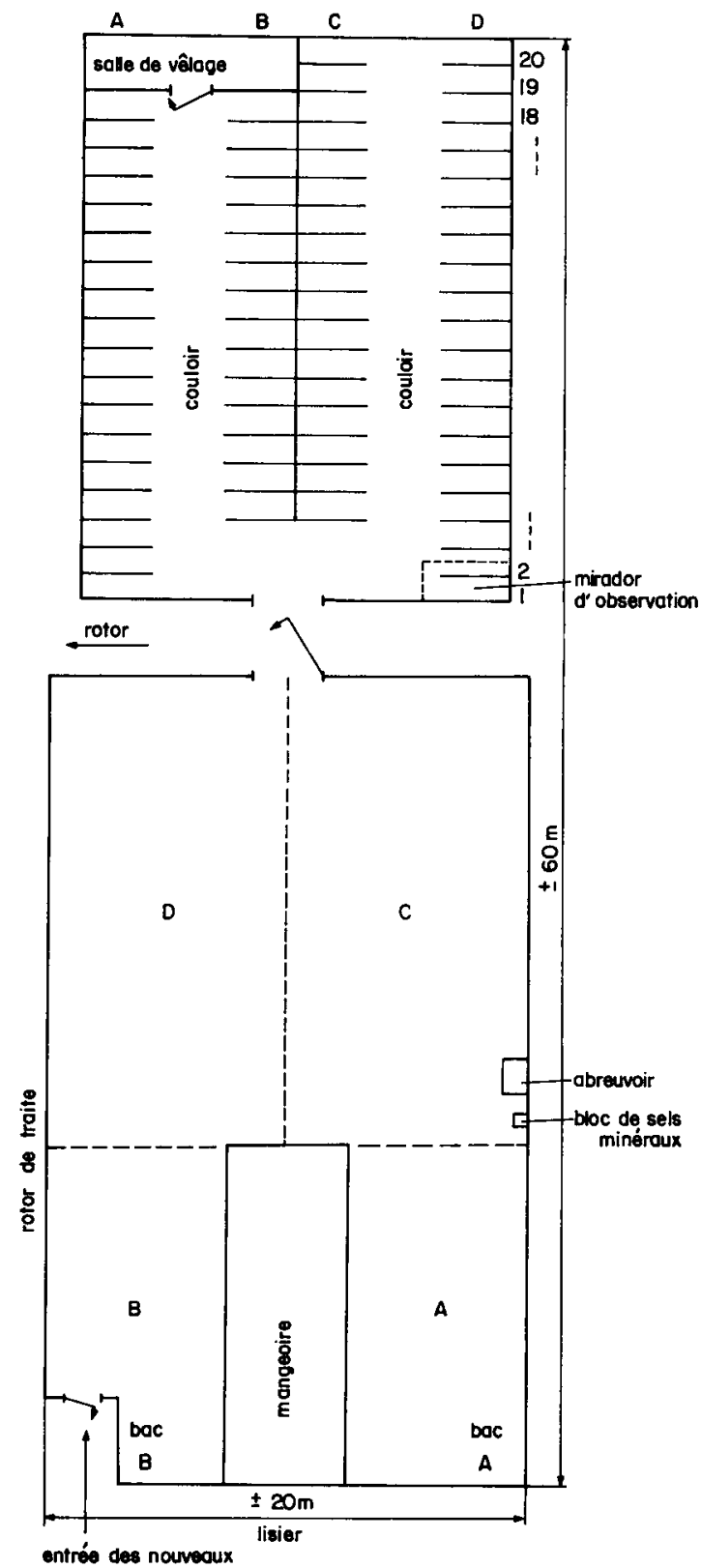

animaux

Fic. I. - Plan du parc extérieur et de l'étable 
mangeoire (fig. I). Les relevés s'effectuaient à partir d'un mirador situé dans l'étable pour les zones $A, C$ et $D$, et d'une fenêtre du rotor de traite pour la zone $B$. Vu le nombre élevé d'animaux et la mobilité de ces derniers, il nous était impossible de noter systématiquement les positions de tous les individus à certains moments. Nous avons cependant pu noter dans quelle aire du parc survenaient les comportements de communication. Une liste de ces derniers a été établie après observation du troupeau. Nous avons dénombré 6 catégories principales. I) Contacts agonistiques : coups avec ou sans effet, écartements à distance et avec contact (1).2) Flairages locaux : tête, cou, flanc, dos, pis, anus, etc. 3) Léchages locaux. 4) Frottements locaux. 5) Contacts simples, c'est-àdire attouchements sans détermination précise mais localisés. 6) Contacts sexuels : contact têtedos, léchage et flairage vaginal suivis ou non de "move" (ScHLOETH, 1958), tentative de monte, érection ct copulation.

Les quatre premiers types de contacts ont été également notés dans des situtions homosexuelles. Ces comportements étaient observés l'après-midi entre $14 \mathrm{~h}$ 30 et $\mathrm{I} 5 \mathrm{~h} 30$. Comme ces derniers n'étaient pas très nombreux ils pouvaient être relevés de façon presque exhaustive (fig. 3). Le fait que deux animaux aient un contact agonistique ou autre dans une des quatre zones a été consiđéré comme un indice de fréquentation de cette dernière. Nous n'avons tenu compte que d'une présence par animal et par zone au cours d'une même période d'observation.

Nous avons également relevé les fréquences de présence dans chacune des deux aires où étaient effectuées les épreuves de nourriture (fig. 4). Ces dernières portaient sur la totalité des animaux et nous ont permis de déterminer la hiérarchie du troupeau (Bourssou et Signoret, I970). Elles étaient effectuées chaque jour successivement dans les deux zones, la nourriture étant donnée alternativement en premier lieu en A ou en B $\left(^{2}\right)$. Le parc était recouvert d'un sol en ciment et la nourriture était distribuée automatiquement une fois par jour dans la mangeoire centrale, celle-ci étant suffisamment grande pour que tous les animaux puissent y manger côte à côte.

\section{RÉSULTATS}

\section{A. - Dans l'étable}

Afin de vérifier si le nombre de présences de chaque animal dans une même stalle était suffisant pour pouvoir rejeter l'hypothèse d'une distribution aléatoire dans l'étable, nous avons calculé la distribution de l'amplitude à partir de la distribution polynomiale qui serait approximativement celle de $\omega_{k} \cdot \sqrt{\mathrm{N} / k}, \omega_{k}$ étant l'amplitude de $k$ variables normales réduites (Young, $\mathbf{1 9 6 2}$ ). Dans notre cas, $k$ étant égal à 70 (nombre de stalles disponibles), en choisissant $\alpha=0,05$, nous trouvons $\omega_{k}=\omega_{70}=$ 5,86 (CADWELL, 1954). Le nombre d'observations (N) variant pour chaque anima1 $\omega_{k} \sqrt{\mathrm{N} / k}$ varie d'un animal à l'autre, $\omega_{k}$ et $k$ étant constants.

Si nous considérons par exemple le taureau, nous avons : $\omega_{k}=5,86, \mathrm{~N}=48$, et l'amplitude maximale observée $=$ Ir. Dès lors $\omega_{k} \sqrt{\mathrm{N} / \bar{k}}=5,86 \sqrt{48 / 70}=4,83$. L'hypothèse d'uniformité de la distribution peut être rejetée dans ce cas étant donné que l'amplitude maximale observée est de II $(>4,83)$.

A l'examen du tableau $I$, on constate que la fréquence d'occupation d'une stalle particulière par un individu donné est systématique. En effet l'ampiitude maximale observée (nombre de fois que l'animal a occupé la même place) est toujours supérieure à l'amplitude calculée, à l'exception de l'animal nº 525 , chez lequel le nombre d'observations est trop faible pour tirer une conclusion statistiquement valable. Les stalles le plus fréquemment occupées par les animaux sont représentées à la figure 2 .

(1) Ln combat véritable précédé de menace et d'approrhe n'a jamais été observé dans notre groupe de vaches laitières.

${ }^{2}$ ) Ce problètue a été traité en détail dans une autre étucle actuellenent en cours de publication. 
TABLEAU I

Distribution de la fréquence d'occupation des stalles

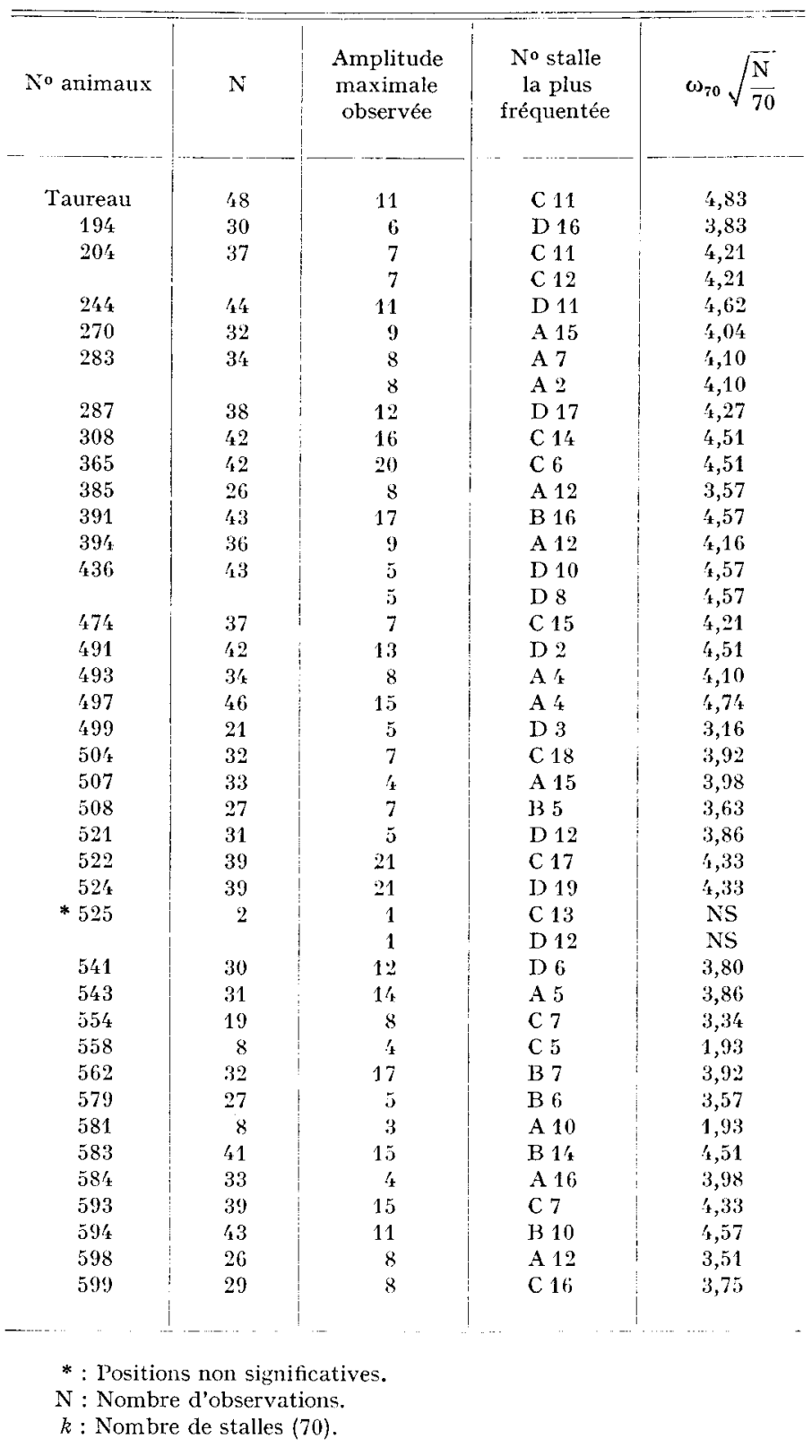


Nous n'avons pas trouvé de corrélation entre le rang social des animaux et le niveau de préférence pour une stalle déterminée $(p>0,80$, épreuve de corrélation de rang de Spearman (Two-tailed SIEger, (I956)).

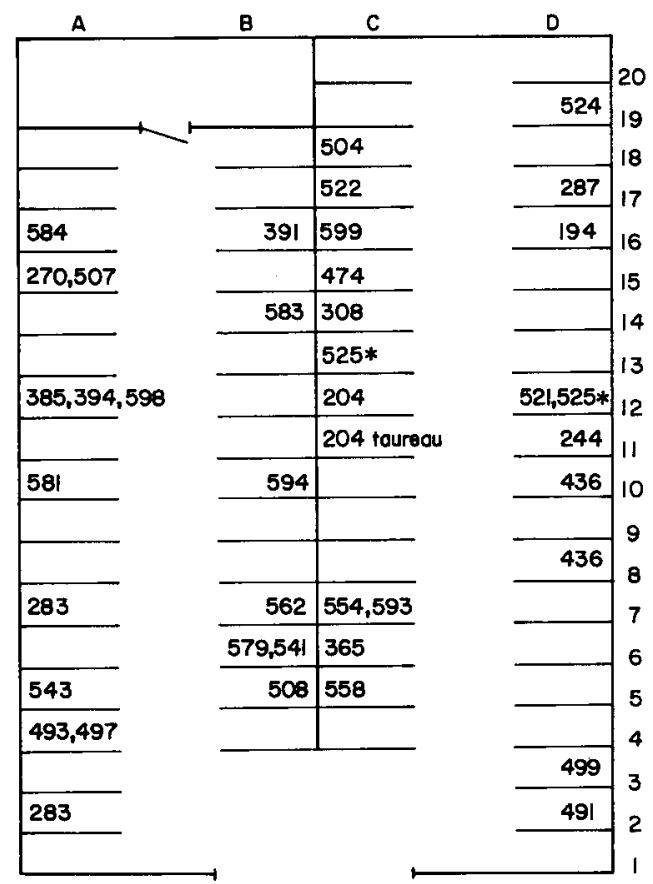

lig. 2. - Stalles les plus fréquentées par les différents animaux à l'intérieur de l'étable L'animal dont le numéro est suivi d'un astérisque (*) ne manifeste pas une préférence statistiquement significative pour la stalle considéréc

\section{B. - Dans le parc cxtérieur}

Étant donné que nous n'avions pas d'hypothèse permettant de prédire le sens des résultats, toutes les épreuves statistiques ont été appliquées dans un sens bidirectionnel (Two-tailed).

I. En ce qui concerne la répartition des animaux sur l'aire totale du parc, nous avons trouvé que l'ensemble du troupeau manifestait une préférence hautement significative pour la zone $\mathrm{B}$ par rapport à chacune des trois autres aires $\mathrm{A}, \mathrm{C}$ et $\mathrm{D}$ ( $p \leqslant 0,000 \mathrm{I}, p \leqslant 0,005$ et $p \leqslant 0,000 \mathrm{OI}$ ) (fig. 3) et que la fréquentation des trois zones cumulées n'était pas significativement plus importante que celle de $B$ seule (test des signes, SrEgEr (I956)). En outre, même en tenant compte du niveau de préférence manifesté par certains animaux pour les zones $\mathrm{A}, \mathrm{C}$ et $\mathrm{D}$, la préférence pour $\mathrm{B}$ reste significative $(p \leqslant 0,000$ or, test de Wilcoxon, SIEGEL (I956)). D'autre part, 5 animaux manifestent une préférence marquée pour une zone par rapport à chacune des trois autres $(p \leqslant 0,05$, test binomial, SIEGEL (I956)).

Enfin, nous avons vérifié s'il existait une relation entre le taux de fréquentation d'une zone et le niveau hiérarchique. Nous avons trouvé que les ro animaux de tête 
étaient repérés significativement plus fréquemment en $\mathrm{C}$ que les Io animaux les plus dominés et que ces derniers étaient observés moins souvent dans les quatre aires cumulées que les Io animaux supérieurs $(p \leqslant 0$,oI test de Mann Withney, SrEGEI. $\left.\left(\mathrm{r} 95^{6}\right)\right)$.
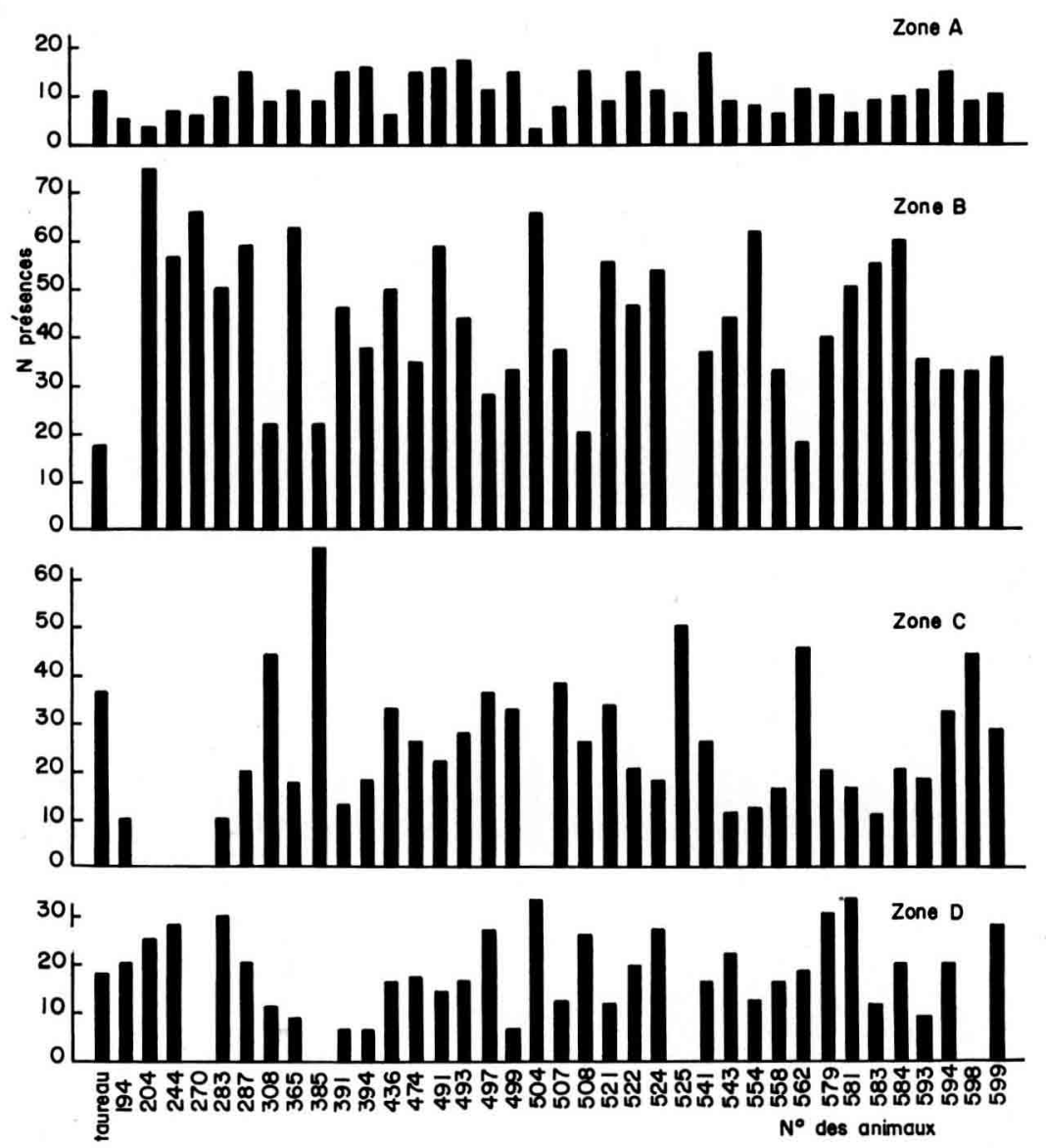

Fig. 3. - Fréquence relative de présence des animaux

dans les quatre zones du parc extérieur en dehors des périodes d'alimentation

Valeurs exprimées en p. Ioo du nombre de présences relevé dans le parc pour chaque animal

2. En ce qui concerne la fréquence de présences dans les zones A et B lors des épreuves de nourriture (fig. 4), nous n'avons trouvé aucune préférence significative de l'ensemble du troupeau pour une des deux aires (test des signes, SigGer (I956)). En considérant chaque animal individuellement, nous remarquons que 8 animaux avaient une préférence marquée pour une des deux zones $(p \leqslant 0,05$ test binomial, SiEgEr, (I956)).

3. Enfin, nous avons voulu examiner si les préférences des animaux pour les deux zones $\mathrm{A}$ et $\mathrm{B}$ étaient identiques dans les deux situations, à savoir lors des épreuves de nourriture et lors des comportements de communication. 

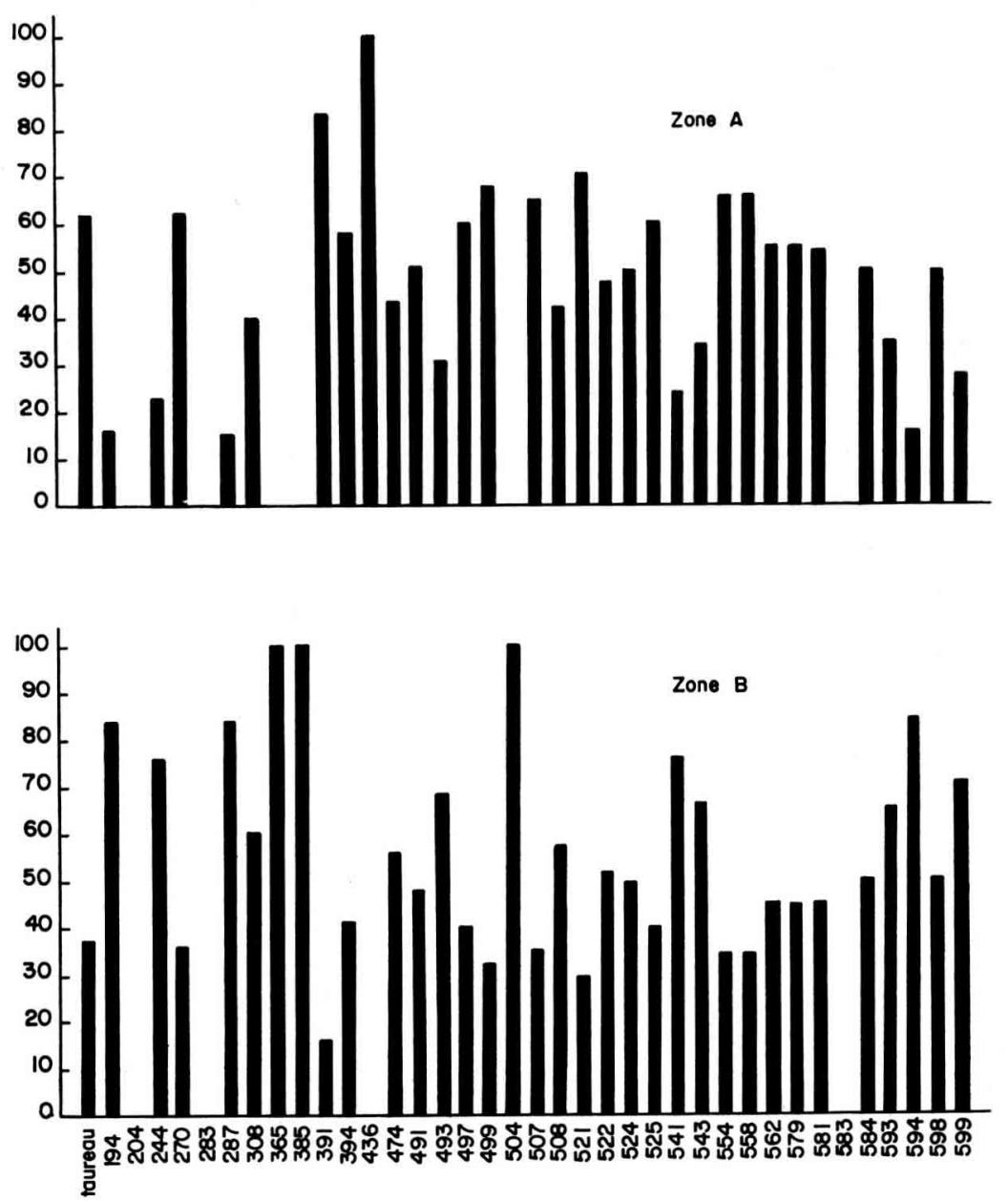

Fig. 4. - Fréquence relative de présence des animaux dans les deux zones d'alimentation Valcurs exprimées en p. Ioo đu nombre de présences relevé dans ces zones pour chaque animal

\section{TABLEAU 2}

Relation entre les zones significativiment plus fréquentées par certains animaux pendant les périodes d'alimentation et en dehors de celles-ci, c'est-à-dire lors des comportements de communication

\begin{tabular}{|c|c|c|c|c|}
\hline $\begin{array}{l}\text { No de } \\
\text { l'animal }\end{array}$ & $\begin{array}{l}\text { Zone préférée } \\
\text { (nourriture) }\end{array}$ & $\begin{array}{l}\text { Niveaux } \\
\text { sign. }\end{array}$ & $\begin{array}{l}\text { Zone préférée } \\
\text { (cpt. commun.) }\end{array}$ & $\begin{array}{l}\text { Niveaux de } \\
\text { sigu. }\end{array}$ \\
\hline$* 19^{\prime}$ & I3 & $p \leqslant 0,05$ & A & NS \\
\hline $2^{\prime} t^{\prime}$ & 13 & $p \leqslant 0,05$ & 13 & NS \\
\hline 287 & $\mathrm{~B}$ & $p \leqslant 0,05$ & B & $p \leqslant 0,05$ \\
\hline 385 & 13 & $p \leqslant 0,05$ & $\mathrm{I} 3$ & NS \\
\hline * 391 & A & $p \leqslant 0,0 j$ & B & NS \\
\hline$* 521$ & A & $p \leqslant 0,05$ & 13 & NS \\
\hline $5 / 1$ & $\mathrm{~J} 3$ & $p \leqslant 0,05$ & $\mathrm{~B}$ & NS \\
\hline 594 & $B$ & $p \leqslant 0,05$ & B & NS \\
\hline
\end{tabular}

* : préférence différente selon le type de situation. 
Nous avons repris au tableau 2 tous les animaux manifestant une préférence significative pour A ou pour B dans l'épreuve de nourriture, et nous remarquons que les résultats trouvés concernant la fréquentation de ces deux zones par les mêmes animaux, en dehors de la distribution de nourriture, ne vont pas nécessairement dans le même sens. Pour certains individus les aires préférentielles sont identiques dans les deux situations, pour d'autres elles sont inversées mais non significativement opposées.

\section{DISCUSSION ET CONCLUSIONS}

Dans ce travail, nous avons utilisé intentionnellement l'expression d'aire de résidence préférentielle plutôt que celle de territoire. L'examen de la littérature montre en effet qu'il existe certaines ambiguités quant au sens qu'il convient d'attribuer au mot " territoire".

Ainsi pour SchLoeth (I96I), la notion de territoire implique uniquement la fréquentation collective de certaines aires particulières liées électivement à certains comportements (territoire de repos, d'alimentation etc.). EIBL-E,IBESFELDT (I970) estime comme Schenker. (Ig66) et ScotT (I969), que le territoire implique l'intolérance d'un individu ou d'un groupe à l'égard d'intrus, et il fait en outre remarquer que la défense de l'aire considérée est souvent un comportement du groupe dans lequel les relations hiérarchiques internes passent au second plan.

Aux yeux des trois derniers auteurs, territoire implique donc indissociablement occupation et défense d'une aire donnée. Comme nos observations, tant dans l'étable que dans le parc extérieur, nous révèlent l'existence d'aires préférentielles, mais ne mettent en évidence aucune défense de celles-ci, nous ne pensons pas pouvoir parler, dans l'état actuel de nos recherches, de territoire au sens qui a été indiqué plus haut. La seule notion univoque qui se dégage donc à propos des bovins que nous avons observés en stabulation libre, est celle d'aire de résidence préférentielle. En raison de son caractère descriptif, ce terme permet d'éviter toute équivoque avec le terme de dominance territoriale ou de territorialité (HEUTS, I968).

En ce qui concerne la répartition des animaux à l'intérieur de l'étable, celle-ci n'étant pas éclairée la nuit, nous avons dî̀ nous contenter de repérer les positions momentanées des animaux. Cependant, il n'est pas exclu que des observations prolongées nous auraient permis de constater des comportements de défense de la zone occupée. Par ailleurs, le fait que chaque animal avait en moyenne deux stalles à sa disposition (38/70) a pu réduire le nombre de compétitions, chacun d'eux disposant au départ de plusieurs possibilités. Il est en effet possible que dans une étable où le nombre de logettes serait égal au nombre d'animaux, les individus hiérarchiquement supérieurs soient favorisés et soient seuls en mesure d'occuper l'espace désiré.

Pour ce qui regarde la constance de position observée dans l'étable, on peut supposer que le repérage de l'aire individuelle serait de nature olfactive, cette modalité sensorielle étant bien développée chez les Bovins (DoRst, I972 ; Bourssou et al., I97I). Quelques observations occasionnelles semblent toutefois montrer que l'olfaction n'est pas seule en cause. En effet, après une absence prolongée, un animal particulier a tendance à venir occuper à nouveau la même stalle. Ce fait a été constaté 
chez une vache $\left(n^{0} 55^{8}\right)$ qui avait été retirée du groupe pendant deux mois au cours de la période d'observation. Avant son vêlage, cet animal avait une préférence marquée pour la stalle $\mathrm{C} 5$ et à son retour il est retourné systématiquement dans cette dernière. D'autre part, lorsque l'on transfère un animal d'une étable à une autre disposée de la même façon, celui-ci occupe dans cette dernière une position homologue de la position antérieure.

Ces observations permettent de supposer qu'il y aurait chez les Bovins une perception configurationnelle de l'espace qu'ils occupent ordinairement. Nous espérons pouvoir vérifier systématiquement cette hypothèse ultérieurement.

Quant aux observations dans le parc extérieur, elles semblent montrer qu'il y existe une aire de prédilection pour les comportements de communication (zone B). Toutefois, nous n'avons trouvé pour aucun animal une différence significative dans les catégories de contacts qui survenaient respectivement dans les quatre zones. Le seul endroit où nous ayons trouvé une proportion nettement plus élevée de contacts agonistiques par rapport au reste du parc, est la mangeoire (pour ro animaux $p \leqslant 0,05$ test binomial, SIEGEL (I956)). Par ailleurs lors des compétitions destinées à établir la hiérarchie, nous avons trouvé que la proportion des victoires et des défaites était identique quel que fût l'endroit du parc où elles se déroulaient (test du $\chi^{2}$ ou test exact de Fisher, Dagneire (I969)).

En conclusion, nos observations semblent indiquer l'existence d'un territoire d'alimentation (mangeoire) mais non de sous-territoires au sein du parc. Cependant, on pourrait considérer l'espace total disponible (parc + étable) comme un territoire collectif, dans la mesure toutefois où des observations mettraient en évidence une défense de cette aire par l'ensemble du groupe.

Reçu pour publication en septombre 1974 .

\title{
REMERCIEMENTS
}

Cette recherche a été subventionnée par l'Institut pour l'encouragement de la Recherche Scientifique dans l'Industric et dans l'Agriculture (I. R. S. I. A.). Nous remercions vivement lo Professeur P. DaGnelie et son collaborateur M. K. IN, de la faculté des sciences agronomiques de l'État à Gembloux dont l'aide nous a été précieuse pour l'analyse statistique des résultats. Notre gratitude s'adresse également au Baron $Y$. de Tokxaco, propriétaire de l'exploitation, sans la collaboration duquel ces observations n'auraient pu être réalisées.

\section{SUMMARY}

\author{
PREFERENTIAL ZONIS VISITED BY DAIRY COWS \\ KEPT IN AN OPEN STAIT, SYSTEM
}

The observations analyzed in the present paper have been carried out on dairy cows kept in an open stall system consisting of an outside cemented area continuously connected with a stable divided in individual boxes, to which the animals had permanent free access. Results obtained on a population comprising 37 cows and $x$ bull, give rise to the following conclusions : 
I. The distribution of animals in the stable is systematic, each animal showing a significant preference for a particular box.

2. The frequentation of the outside area is fairly homogeneous. However, a particular zone is more often occupied by the whole of the herd and some animals show marked local preferences. One may suppose that the choice of preferential boxes is guided partly by a configurational perception of the available area, though it is not possible to establish, on the basis of the data collected up to now, which sensory signals may be responsible for this behaviour. In the outside area, there exists a preferential zone linked with communication behaviour patterns and a feeding territory. The legitimacy of the notion of territory is discussed, in relation with the observed spatial distributions.

\section{RÉFÉRENCESS BIBLIOGRAPHIQUES}

Beilharz R. G., Mylrea P. J., I963. Social position and behaviour of dairy heifers in yard. Anim. Behav., 11, 522-528.

Bouissou M. F., Srgnoret J.-P., 1970. La hiérarchie sociale chez les Mammifères. Rev. Comp. Animal, 4, 43-6I.

Bouissou M. F., Rouger Y., Signoret J.-P., rg7i. Intercommunications chez les ongulés. J.Psychol. Norm. et Pathol., 34, 4I 5-43I.

CADWELL J. H., I954. The probability integral of range for sample from a symmetrical unimodal population. Ann. Math. Statist., 25, 803-806.

Dagnelie P., r969. Théorie et méthodes statistiques, II, Gembloux, Duculot.

Dorst J., 1972. Les chimiorécepteurs. In : P. P. Grassḱ, Traité de Zoologie, vol. 16 (4), 418-482, Paris, Masson.

Eibl-Eibesfeidt, 1970. Ethology, the biology of behaviour, N. Y. Holt, Rinehart and Wilson.

Hancock J., r953. Grazing behaviour of cattle. Anim. Breed. Abstr., 21, I-I3.

Heuts B. A., 1968. A presumed case of territoriality in Poeciliids (Pisces, Cyprinodontiformes). Rev. Can. Biol., 27, 297-3r2.

Schenkel R., 1966. Zum Problem der Territoralität und des Markierens bei Säugern am Beispiel des Schwarzen Nashorns und Löwen. Z. Tierpsychol., 23, 593-626.

Schloeti R., I958. Cycle annuel et comportement social du taureau de Camargue. Mammalia, 22, I2I-I39.

Schloeth R., I96r. Das Sozialleben des Camargue-rindes. Z. Tierpsychol., 18, 574-627.

Scotт J.-P., I969. Introduction to animal behaviour. In : The Behaviour of Domestic Animals. (E. HAFEZ, ed.), 3-21. London, Baillière, 'Tindall and Cassel.

SiEgEL S., I956. Non parametric Statistics for the behavioral Sciences. N. Y. Mc. Graw-Hill.

Tribe D. F., I955. The behaviour of grazing animals. In : Progress in the Physiology of Famm Animals (L. Hammond, ed.) Vol. 2, 585. London, Butterworths.

Vorsin A., I959. Grass Productivity. New York, N. Y., Philosophical Library.

Young D. H., I962. Two alternatives to the standard $\chi^{2}$ test of the hypothesis of equal cell frequenties. Biometrica, $49,107-\mathrm{r} \pm 6$. 